\title{
Research Article \\ Effects of Magnetic Field and an Endoscope on Peristaltic Motion
}

\author{
V. P. Rathod and S. K. Asha \\ Department of Studies and Research in Mathematics, Gulbarga University, Gulbarga 585106, \\ Karnataka, India \\ Correspondence should be addressed to S. K. Asha, as.kotnur2008@gmail.com
}

Received 25 November 2010; Accepted 20 January 2011

Academic Editor: F. Marcellan

Copyright (C) 2011 V. P. Rathod and S. K. Asha. This is an open access article distributed under the Creative Commons Attribution License, which permits unrestricted use, distribution, and reproduction in any medium, provided the original work is properly cited.

The Problem of peristaltic transport of a magnetic fluid with variable viscosity through the gap between coaxial tubes where the outer tube is nonuniform with sinusoidal wave traveling down its wall and the inner tube is rigid. The relation between the pressure gradient and friction force on the inner and outer tubes is obtained in terms of magnetic and viscosity parameter. The numerical solutions of pressure gradient, outer friction and inner friction force, and flow rate are shown graphically.

\section{Introduction}

The purpose of this paper is an attempt to understand the fluid mechanics in a physiological situation with the presence of an endoscope placed concentrically. The pressure rise, peristaltic pumping, augmented pumping, and friction force on the inner tube (endoscope) and outer tube are discussed by Srivastava et al. [1] and Siddiqui and Schwarz [2]. Latham [3] investigated the fluid mechanics of peristaltic pump and since then, other work on the same subject has been followed by Burns and Parkes [4]. Barton and Raynor [5] have been studied the case of a vanishingly small Reynolds number. Lykoudis and Roos [6] studied the fluid mechanics of the ureter from a lubrication theory point of view. Zien and Ostrach [7] have investigated a long wave approximation to peristaltic motion, and the analysis is aimed at the possible application to urine flow in human ureters. Roos and Lykoudis [8] studied the effect of the presence of a catheter upon the pressure distribution inside the ureter. Ramchandra and Usha [9] studied the influence of an eccentrically inserted catheter on the peristaltic pumping in a tube under long wavelength and low Reynolds numbers approximations. Abd El Naby and El Misery [10] studied the effect of an endoscope and generalized Newtonian fluid on 
peristaltic motion. Gupta and Sheshadri [11] studied peristaltic transport of a Newtonian fluid in nonuniform geometries. L. M. Srivastava and V. P. Srivastava [12] have investigated the effect of power law fluid in uniform and nonuniform tube and channel under zero Reynolds number and long wavelength approximation. Provost and Schwarz [13] have investigated a theoretical study of viscous effect in peristaltic pumping and assumed that the flow is free of inertial effect and that non-Newtonian normal stresses are negligible. Boehme and Friedrich [14] have investigated peristaltic flow of viscoelastic liquids and assumed that the relevant Reynolds number is small enough to neglect inertia forces, and that the ratio of the wavelength and channel height is large, which implies that the pressure is constant over the cross section. El Misery et al. [15] have investigated the effect of a Carreau fluid in peristaltic transport for uniform channel. Elshehaway et al. [16] studied peristaltic motion of generalized Newtonian fluid in a nonuniform channel under zero Reynolds number with long wavelength approximation. Most of studies on peristaltic motion, that assume physiological fluids behave like a Newtonian fluid with constant viscosity, fail to give a better understanding when peristaltic mechanics is involved in small blood vessel, lymphatic vessel, intestine, ducts efferent of the male reproductive tracts, and in transport of spermatozoa in the cervical canal. According to Haynes [17], Bugliarllo and Sevilla [18] and Goldsmith and Skalak [19], it is clear that in prementioned body organs, viscosity of the fluid varies across the thickness of the duct. Cotton and Williams [20] study the practical gastrointestinal endoscope. Rathod and Asha [21] studied the peristaltic transport of a couple stress fluids in uniform and nonuniform annulus moving with a constant velocity. Rathod and Asha [22] studied the effect of couple stress fluid and an endoscope on peristaltic motion.

In the view of above discussion, the effect of magnetic fluid with variable viscosity through the gap between inner and outer tubes where the inner tube is an endoscope and the outer tube has a sinusoidal wave traveling down its wall is the aim of present investigation.

\section{Formulation and Analysis}

Consider the two-dimensional flow of an incompressible Newtonian fluid with variable viscosity through the gap between inner and outer tubes where the inner tube is an endoscope and the outer tube has a sinusoidal wave traveling down its wall. The geometry of the two wall surface is given by the equation:

$$
\begin{gathered}
\overline{r_{1}}=a_{1}, \\
\overline{r_{1}}=a_{20}+b \sin \frac{2 \pi}{\lambda}(\bar{z}-c \bar{t}),
\end{gathered}
$$

where $a_{1}$ is the radius of endoscope, $a_{20}$ is the radius of the small intestine at inlet, $b$ is the amplitude of the wave, $\lambda$ is the wavelength, $\bar{t}$ is time, and $c$ is the wave speed.

In the fixed coordinates $(\bar{r}, \bar{z})$, the flow in the gap between inner and outer tubes is unsteady but if we choose moving coordinates $(\bar{r}, \bar{z})$ which travel in the $\bar{z}$-direction with the same speed as the wave, then the flow can be treated as steady. The coordinate's frames are related through

$$
\begin{aligned}
\bar{z}=\bar{Z}-c \bar{t}, & \bar{r}=\bar{R}, \\
\bar{w}=\bar{W}-c, & \bar{u}=\bar{U},
\end{aligned}
$$


where $\bar{U}, \bar{W}$ and $\bar{u}, \bar{w}$ are the velocity components in the radial and axial direction in the fixed and moving coordinates, respectively.

Equations of boundary condition in the moving coordinates are continuity equation:

$$
\frac{1}{\bar{r}} \frac{\delta(\bar{r} \bar{u})}{\delta \bar{r}}+\frac{\delta \bar{w}}{\delta \bar{z}}=0
$$

and the Navier Stokes equation:

$$
\begin{aligned}
& \rho\left(\bar{u} \frac{\partial \bar{u}}{\partial \bar{r}}+\bar{w} \frac{\partial \bar{u}}{\partial \bar{z}}\right)=\frac{\partial \bar{p}}{\partial \bar{r}}+\frac{\partial}{\partial \bar{r}}\left[2 \bar{\mu}(\bar{r}) \frac{\partial \bar{u}}{\partial \bar{r}}\right]+\frac{2 \bar{\mu}(\bar{r})}{\bar{r}}\left(\frac{\partial \bar{u}}{\partial \bar{r}}-\frac{\bar{u}}{\bar{r}}\right)+\frac{\partial}{\partial \bar{z}}\left[\bar{\mu}(\bar{r})\left(\frac{\partial \bar{u}}{\partial \bar{z}}+\frac{\partial \bar{w}}{\partial \bar{r}}\right)\right]-\sigma B_{0}^{2}(\bar{u}), \\
& \rho\left(\bar{u} \frac{\partial \bar{w}}{\partial \bar{r}}+\bar{w} \frac{\partial \bar{w}}{\partial \bar{z}}\right)=-\frac{\partial \bar{p}}{\partial \bar{z}}+\frac{\partial}{\partial \bar{z}}\left[2 \bar{\mu}(\bar{r}) \frac{\partial \bar{w}}{\partial \bar{z}}\right]+\frac{1}{\bar{r}} \frac{\partial}{\partial \bar{r}}\left[\bar{\mu}(\bar{r}) \bar{r}\left(\frac{\partial \bar{u}}{\partial \bar{z}}+\frac{\partial \bar{w}}{\partial \bar{r}}\right)\right]-\sigma B_{0}^{2}(\bar{w}) .
\end{aligned}
$$

$\bar{p}$ is the pressure, $\bar{\mu}(\bar{r})$ is the viscosity function, $\sigma$ is Electric conductivity, and $B_{0}$ is applied magnetic field. The boundary conditions are written as follows:

$$
\begin{gathered}
\bar{w}=-c \quad \text { at } u=0, \bar{r}=\bar{r}_{1}, \bar{r}=\bar{r}_{2}, \\
\bar{u}=0 \quad \text { at } \bar{r}=\bar{r}_{1} .
\end{gathered}
$$

We introduce the nondimensional variable and the Reynolds number (Re) and the wave number $(\delta)$ introduced.

$$
\begin{gathered}
r=\frac{\bar{r}}{a_{20}}, \quad R=\frac{\bar{R}}{a_{20}}, \quad r_{1}=\frac{\bar{r}_{1}}{a_{20}}=\frac{a_{10}}{a_{20}}=\varepsilon<1, \quad z=\frac{\bar{z}}{\lambda}, \quad Z=\frac{\bar{Z}}{\lambda}, \\
\mu(r)=\frac{\bar{\mu}(\bar{r})}{\mu_{0}}, \quad u=\frac{\lambda \bar{u}}{a_{20} c}, \quad U=\frac{\lambda \bar{U}}{a_{20} c}, \quad w=\frac{\bar{w}}{c}, \quad W=\frac{\bar{W}}{c}, \quad \delta=\frac{a_{20}}{\lambda} \ll 1, \\
\operatorname{Re}=\frac{c a_{20} \rho}{\mu_{0}}, \quad p=\frac{a_{20}^{2} \bar{p}}{c \lambda \mu_{0}}, \quad t=\frac{c \bar{t}}{\lambda}, \quad \phi=\frac{b}{a_{20}}<1, \\
r_{2}=\frac{\bar{r}_{2}}{a_{20}}=1+\phi \sin 2 \pi z,
\end{gathered}
$$


where $\varepsilon$ is the radius ratio, $\phi$ is the amplitude ratio, and $\mu_{0}$ is the viscosity on the endoscope. Equation of motion and boundary conditions in the dimensionless form become

$$
\begin{aligned}
\frac{1}{r} \frac{\partial(r u)}{\partial r}+\frac{\partial w}{\partial z}= & 0 \\
\operatorname{Re} \delta^{3}\left(u \frac{\partial u}{\partial r}+w \frac{\partial u}{\partial z}\right)= & -\frac{\partial p}{\partial r}+\delta^{2} \frac{\partial}{\partial r}\left(2 \mu(r) \frac{\partial u}{\partial r}\right)+\delta^{2} \frac{\partial}{\partial z}\left[\mu(r)\left(\delta^{2} \frac{\partial u}{\partial z}+\frac{\partial w}{\partial r}\right)\right] \\
& +\frac{2 \delta^{2} \mu(r)}{r}\left(\frac{\partial u}{\partial r}-\frac{u}{r}\right)-\delta^{2} M^{2}(u) \\
\operatorname{Re} \delta\left(u \frac{\partial w}{\partial r}+w \frac{\partial w}{\partial z}\right)= & -\frac{\partial p}{\partial z}+\frac{1}{r} \frac{\partial}{\partial r}\left[\mu(r) r\left(\delta^{2} \frac{\partial u}{\partial z}+\frac{\partial w}{\partial r}\right)\right] \\
& +\delta^{2} \frac{\partial}{\partial z}\left(2 \mu(r) \frac{\partial w}{\partial z}\right)-M^{2}(w)
\end{aligned}
$$

$M=\sqrt{\sigma / \mu} B_{0} a_{20}$ is Hartmann number and $\sigma$ is Electric conductivity With the dimensionless boundary condition,

$$
\begin{gathered}
w=-1, \quad u=0, \quad \text { at } r=r_{1}, r=r_{2}, \\
u=0, \quad \text { at } r=r_{1} .
\end{gathered}
$$

Using the long wavelength approximation and neglecting the wave number $(\delta=0)$, one can reduce Navier-Stokes equation

$$
\begin{aligned}
& \frac{\partial p}{\partial r}=0 \\
& \frac{\partial p}{\partial z}=\frac{1}{r} \frac{\partial}{\partial r}\left(\mu(r) r \frac{\partial w}{\partial r}\right)-M^{2}(w) .
\end{aligned}
$$

The instantaneous volume flow rate in the fixed coordinate system is given by

$$
\bar{Q}=2 \pi \int_{\bar{r}_{1}}^{\bar{r}_{2}} \overline{W R} d \bar{R}
$$

where $\bar{r}_{1}$ is a constant and $\bar{r}_{2}$ is a function of $\bar{Z}$ and $\bar{t}$. On substituting (2.3) into (2.11) and the integrating, one obtains

$$
\bar{Q}=\bar{q}+\pi c\left(\bar{r}_{2}^{2}-\bar{r}_{1}^{2}\right)
$$

where

$$
\bar{q}=2 \pi \int_{\bar{r}_{1}}^{\bar{r}_{2}} \bar{w} \bar{r} d \bar{r}
$$


Is the volume flow rate in the moving coordinate system and is it independent of time? Here, $\bar{r}_{2}$ is a function of $\bar{z}$ alone and is defined through (2.2). Using the dimensionless variable, we find that (2.13) becomes

$$
F=\frac{\bar{q}}{2 \pi a_{20}^{2} c}=\int_{r_{1}}^{r_{2}} w r d r
$$

The time-mean flow over a period $T=\lambda / c$ at a fixed $\mathrm{Z}$ position is defined as

$$
Q=\frac{1}{T} \int_{0}^{T} \bar{Q} d \bar{t}
$$

Using (2.12) and (2.13) in (2.15) and integrating, we get

$$
\bar{Q}=\bar{q}+\pi c\left(a_{2}^{2}-a_{1}^{2}+\frac{b^{2}}{2}\right),
$$

which may be written as

$$
\frac{\bar{Q}}{2 \pi a_{20}^{2} c}=\frac{\bar{q}}{2 \pi a_{20}^{2} c}+\frac{1}{2}\left(1-\varepsilon^{2}+\frac{\phi^{2}}{2}\right) .
$$

On defining the dimensionless time-mean flow as

$$
\Theta=\frac{\bar{Q}}{2 \pi a_{20}^{2} c},
$$

writing (2.17) as

$$
\Theta=F+\frac{1}{2}\left(1-\varepsilon^{2}+\frac{\phi^{2}}{2}\right)
$$

and solving (2.9)-(2.10), we obtain

$$
W=\frac{1}{2} \frac{d p}{d z}\left[I_{1}(r)-\frac{I_{2}(r)\left\{I_{1}\left(r_{2}\right)-I_{1}\left(r_{1}\right)\right\}-I_{1}\left(r_{1}\right) I_{2}\left(r_{2}\right)-I_{2}\left(r_{1}\right) I_{1}\left(r_{2}\right)}{I_{2}\left(r_{2}\right)-I_{2}\left(r_{1}\right)}\right]-\left(\frac{1}{2}\right) M^{2}(D)-1,
$$

where $D=\left(I_{2}(r)\left\{I_{1}\left(r_{2}\right)-I_{1}\left(r_{1}\right)\right\}-I_{1}\left(r_{1}\right) I_{2}\left(r_{2}\right)-I_{2}\left(r_{1}\right) I_{1}\left(r_{2}\right)\right) /\left(I_{2}\left(r_{2}\right)-I_{2}\left(r_{1}\right)\right)$,

$$
\begin{aligned}
& I_{1}(r)=\int \frac{r}{\mu(r)} d r, \\
& I_{2}(r)=\int \frac{d r}{r \mu(r)} .
\end{aligned}
$$


Using (2.14) we obtain the relationship between $d p / d z$ and $F$ as follows:

$$
\begin{gathered}
F=\frac{1}{4} \frac{d p}{d z}\left[\frac{\left(I_{1}\left(r_{2}\right)-I_{1}\left(r_{1}\right)\right)^{2}}{I_{2}\left(r_{2}\right)-I_{2}\left(r_{1}\right)}-I_{3}\right]+\frac{1}{4} M^{2}\left[\frac{\left(I_{1}\left(r_{2}\right)-I_{1}\left(r_{1}\right)\right)^{2}}{I_{2}\left(r_{2}\right)-I_{2}\left(r_{1}\right)}-I_{3}\right]-\frac{1}{2}\left(r_{2}^{2}-r_{1}^{2}\right), \\
I_{3}=\int_{r_{1}}^{r_{2}} \frac{r^{3}}{\mu(r)} d r .
\end{gathered}
$$

Solving (2.22) for $d p / d z$, we obtain

$$
\frac{d p}{d z}=\frac{4 F+2\left(r_{2}^{2}-r_{1}^{2}\right)-(1 / 4) M^{2}\left(\left[I_{1}\left(r_{2}\right)-I_{1}\left(r_{1}\right)\right]^{2} /\left(I_{2}\left(r_{2}\right)-I_{2}\left(r_{1}\right)\right)-I_{3}\right)}{\left[I_{1}\left(r_{2}\right)-I_{1}\left(r_{1}\right)\right]^{2} /\left(I_{2}\left(r_{2}\right)-I_{2}\left(r_{1}\right)\right)-I_{3}}
$$

The pressure rise $\Delta P_{\lambda}$ and friction force on inner and outer tubes $F_{\lambda}^{(i)}$ and $F_{\lambda}^{(o)}$, in their nondimensional forms, are given by

$$
\begin{aligned}
& \Delta P_{\lambda}=\int_{0}^{1}\left(\frac{d p}{d z}\right) d z \\
& F_{\lambda}^{(i)}=\int_{0}^{1} r_{1}^{2}\left(-\frac{d p}{d z}\right) d z \\
& F_{\lambda}^{(o)}=\int_{0}^{1} r_{2}^{2}\left(-\frac{d p}{d z}\right) d z
\end{aligned}
$$

The effect of viscosity variation on peristaltic transport can be investigated through (2.25) for any given viscosity function $\mu(r)$.

For the present instigation, we assume viscosity variation in the dimensionless form following Srivastava et al. [1] as follows:

$$
\mu(r)=e^{-a r}
$$

or

$$
\mu(r)=1-\alpha r \quad \text { for } \alpha \ll 1
$$

where $\alpha$ is viscosity parameter. The assumption is reasonable for the following physiological reason. Since a normal person of animal or similar size takes 1 to $2 \mathrm{~L}$ of fluid every day, another 6 to $7 \mathrm{~L}$ of fluid are received by the small intestine daily as secretion from salivary glands, stomach, pancreas, liver, and the small intestine itself. This implies that concentration of fluid is dependent on the radial distance. Therefore, the above choice of $\mu(r)=e^{-a r}$ is justified. 
Journal of Applied Mathematics

Substituting (2.27) into (2.21), and (2.23), and using (2.24), we obtain

$$
\begin{aligned}
\frac{d p}{d z}= & {\left[\left\{16 \Theta-8\left(1-\varepsilon^{2}+\left(\phi^{2} / 2\right)\right)+8\left(r_{2}^{2}-r_{1}^{2}\right)\right\} /\left\{\frac{\left(r_{2}^{2}-r_{1}^{2}\right)^{2}}{\log \left(r_{2} / r_{1}\right)}-\left(r_{2}^{4}-r_{1}^{4}\right)\right\}\right] } \\
& \times\left(1-\left(4 \alpha+M^{2}\right)\left\{\frac{\left(r_{2}^{2}-r_{1}^{2}\right)\left(r_{2}^{3}-r_{1}^{3}\right)}{3 \log \left(r_{2} / r_{1}\right)}-\frac{\left(r_{2}^{2}-r_{1}^{2}\right)^{2}\left(r_{2}-r_{1}\right)}{4\left(\ln \left(r_{2} / r_{1}\right)\right)^{2}}-\frac{\left(r_{2}^{5}-r_{1}^{5}\right)}{5}\right\} /\right. \\
& \left.\left\{\frac{\left(r_{2}^{2}-r_{1}^{2}\right)^{2}}{\log \left(r_{2} / r_{1}\right)}-\left(r_{2}^{4}-r_{1}^{4}\right)\right\}\right) .
\end{aligned}
$$

Substituting (2.28) in (2.25) yield

$$
\begin{aligned}
& \Delta P_{\lambda}= \int_{0}^{1}\left[\left\{16 \Theta-8\left(1-\varepsilon^{2}+\phi^{2} / 2\right)+8\left(r_{2}^{2}-r_{1}^{2}\right)\right\} /\left\{\frac{\left(r_{2}^{2}-r_{1}^{2}\right)^{2}}{\log \left(r_{2} / r_{1}\right)}-\left(r_{2}^{4}-r_{1}^{4}\right)\right\}\right] \\
& \times\left(1-\left(4 \alpha+M^{2}\right)\left\{\frac{\left(r_{2}^{2}-r_{1}^{2}\right)\left(r_{2}^{3}-r_{1}^{3}\right)}{3 \log \left(r_{2} / r_{1}\right)}-\frac{\left(r_{2}^{2}-r_{1}^{2}\right)^{2}\left(r_{2}-r_{1}\right)}{4\left(\log \left(r_{2} / r_{1}\right)\right)^{2}}-\frac{\left(r_{2}^{5}-r_{1}^{5}\right)}{5}\right\} /\right. \\
&\left.\left\{\frac{\left(r_{2}^{2}-r_{1}^{2}\right)^{2}}{\log \left(r_{2} / r_{1}\right)}-\left(r_{2}^{4}-r_{1}^{4}\right)\right\}\right) d z, \\
& F_{\lambda}^{(i)}=\int_{0}^{1}-r_{1}^{2}\left[\left\{16 \Theta-8\left(1-\varepsilon^{2}+\phi^{2} / 2\right)+8\left(r_{2}^{2}-r_{1}^{2}\right)\right\} /\left\{\frac{\left(r_{2}^{2}-r_{1}^{2}\right)^{2}}{\log \left(r_{2} / r_{1}\right)}-\left(r_{2}^{4}-r_{1}^{4}\right)\right\}\right. \\
& \times\left(1-\left(4 \alpha+M^{2}\right)\left\{\frac{\left(r_{2}^{2}-r_{1}^{2}\right)\left(r_{2}^{3}-r_{1}^{3}\right)}{3 \log \left(r_{2} / r_{1}\right)}-\frac{\left(r_{2}^{2}-r_{1}^{2}\right)^{2}\left(r_{2}-r_{1}\right)}{4\left(\log \left(r_{2} / r_{1}\right)\right)^{2}}-\frac{\left(r_{2}^{5}-r_{1}^{5}\right)}{5}\right\} /\right. \\
&\left.\left.\left\{\frac{\left(r_{2}^{2}-r_{1}^{2}\right)^{2}}{\log \left(r_{2} / r_{1}\right)}-\left(r_{2}^{4}-r_{1}^{4}\right)\right\}\right)\right] d z,
\end{aligned}
$$




$$
\begin{aligned}
F_{\lambda}^{(o)}= & \int_{0}^{1}-r_{2}^{2}\left[\left\{16 \Theta-8\left(1-\varepsilon^{2}+\left(\phi^{2} / 2\right)\right)+8\left(r_{2}^{2}-r_{1}^{2}\right)\right\} /\left\{\frac{\left(r_{2}^{2}-r_{1}^{2}\right)^{2}}{\log \left(r_{2} / r_{1}\right)}-\left(r_{2}^{4}-r_{1}^{4}\right)\right\}\right. \\
& \times\left(1-\left(4 \alpha+M^{2}\right)\left\{\frac{\left(r_{2}^{2}-r_{1}^{2}\right)\left(r_{2}^{3}-r_{1}^{3}\right)}{3 \log \left(r_{2} / r_{1}\right)}-\frac{\left(r_{2}^{2}-r_{1}^{2}\right)^{2}\left(r_{2}-r_{1}\right)}{4\left(\log \left(r_{2} / r_{1}\right)\right)^{2}}-\frac{\left(r_{2}^{5}-r_{1}^{5}\right)}{5}\right\} /\right. \\
& \left.\left.\left\{\frac{\left(r_{2}^{2}-r_{1}^{2}\right)^{2}}{\log \left(r_{2} / r_{1}\right)}-\left(r_{2}^{4}-r_{1}^{4}\right)\right\}\right)\right] d z .
\end{aligned}
$$

\section{Results and Discussions}

The dimensionless pressure rise $\left(P_{\lambda}\right)$ and the friction forces on the inner and outer tube for different given values of the dimensionless flow rate $\Theta$, amplitude ratio $\phi$, radius ratio $\varepsilon$, Hartmann number $M$, and viscosity parameter $\alpha$ are computed using the (2.29) to (2.31). As the integrals in (2.29) to (2.31) are not integrable in the closed form so they are evaluated using

$$
a_{20}=1.25 \mathrm{~cm}, \quad \frac{a}{\lambda}=0.156
$$

The values of viscosity parameter $\alpha$ as reported by Srivastava et al. [1] are $\alpha=0.0$ and $\alpha=0.1$. Furthermore, since most routine upper gastrointestinal endoscopes are between 8 $11 \mathrm{~mm}$ in diameter as reported by Cotton and Williams [20] and radius ratio $1.25 \mathrm{~cm}$ reported by L. M. Srivastava and V. P. Srivastava [12].

Figure 1 Shows the pressure rise against the flow rate; here it is observed that the pressure increases with the increase of flow rate for different values of radius ratio $\varepsilon=$ $0.32, \varepsilon=0.38$, and $\varepsilon=0.44$ and pressure decreases for the viscosity $\alpha=0.0$ and $\alpha=0.1$. Figure 2 shows that as the viscosity $\alpha$ increases the pressure decreases. And for the different values of amplitude ratio $\phi=0.0$ and $\phi=0.4$, the pressure decreases.

Figures 3 and 4 show the friction force on the outer tube for different values of radius ratio and amplitude ratio; here it is observed that as radius ratio increases the friction force also decreases and they are independent of radius ratio at certain values of the flow rate (for the values $\varphi=0.4$ and $\alpha=0.0$ and $\alpha=0.1$ ). In Figures 5 and 6 , it is noticed that the friction force on the inner tube (endoscope) and on outer tube is plotted against the flow rate for different values of amplitude ratio $\varphi$ and for different values radius ratio $\varepsilon=0.32$, $\varepsilon=0.38$, and $\varepsilon=0.44$ and for the values of viscosity $\alpha=0.0$ and $\alpha=0.1$. It is noticed that as the amplitude ratio $\varphi$ increases the friction force on the outer tube and inner tube decreases and as the viscosity increases the friction force on the outer tube and inner tube decreases.

From Figure 7, it is noticed that the pressure increases for different values of magnetic field $M=1,3$, and 5. From Figures 8 and 9, it is noticed that the friction force decreases on endoscope and on the outer tube as magnetic field increases. 


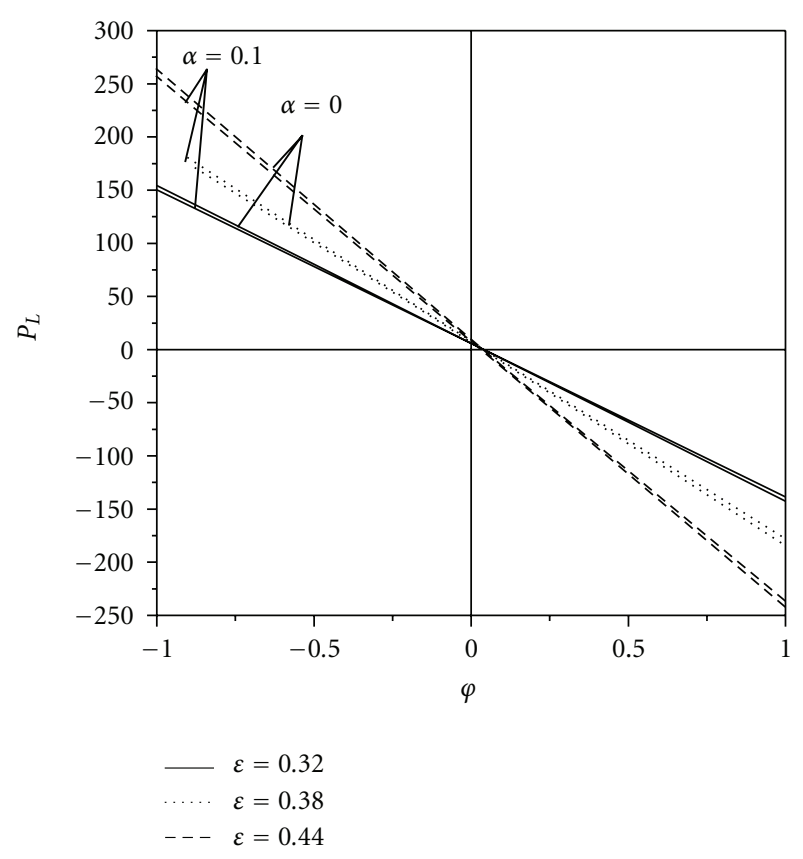

Figure 1: The pressure rise versus flow rate for $\phi=0.4$ and $\alpha=0.0$ and $\alpha=0.1$.

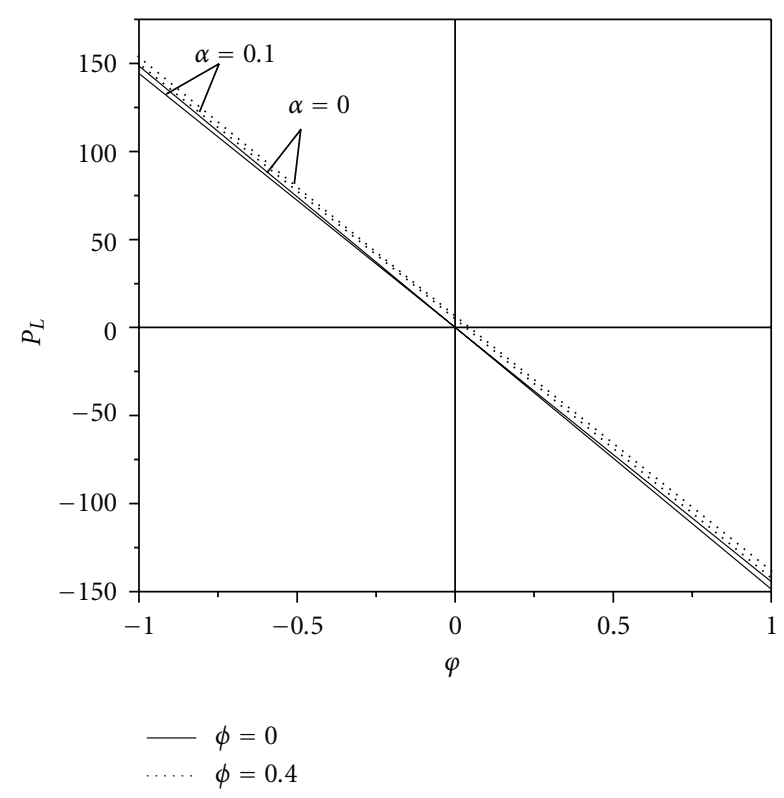

Figure 2: The pressure rise versus flow rate for $\varepsilon=0.32, \alpha=0.0$, and $\alpha=0.1$. 


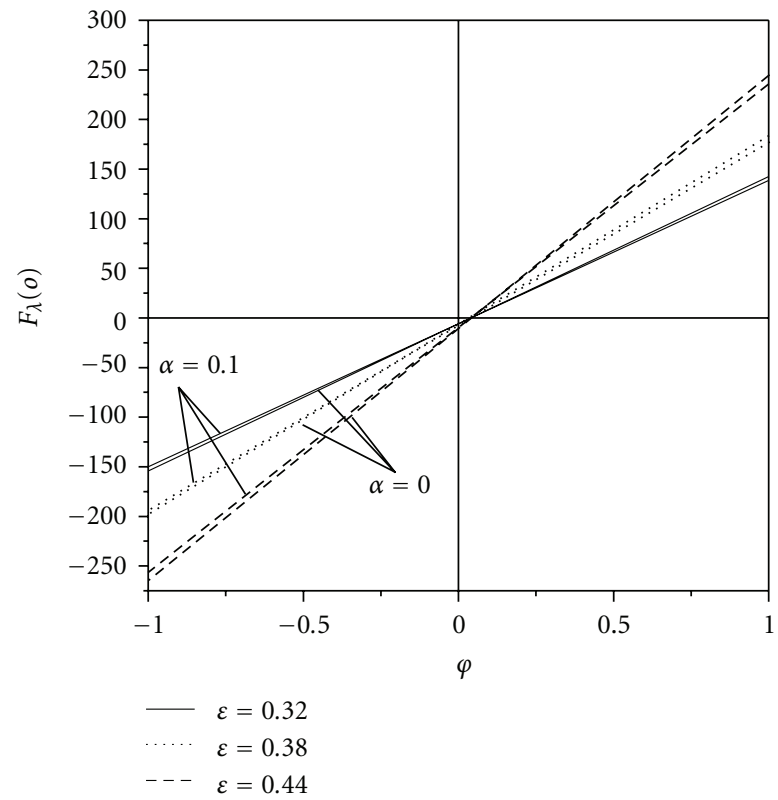

Figure 3: The friction force on the outer tube versus flow rate for $\phi=0.2$ and $\alpha=0.0, \alpha=0.1$.

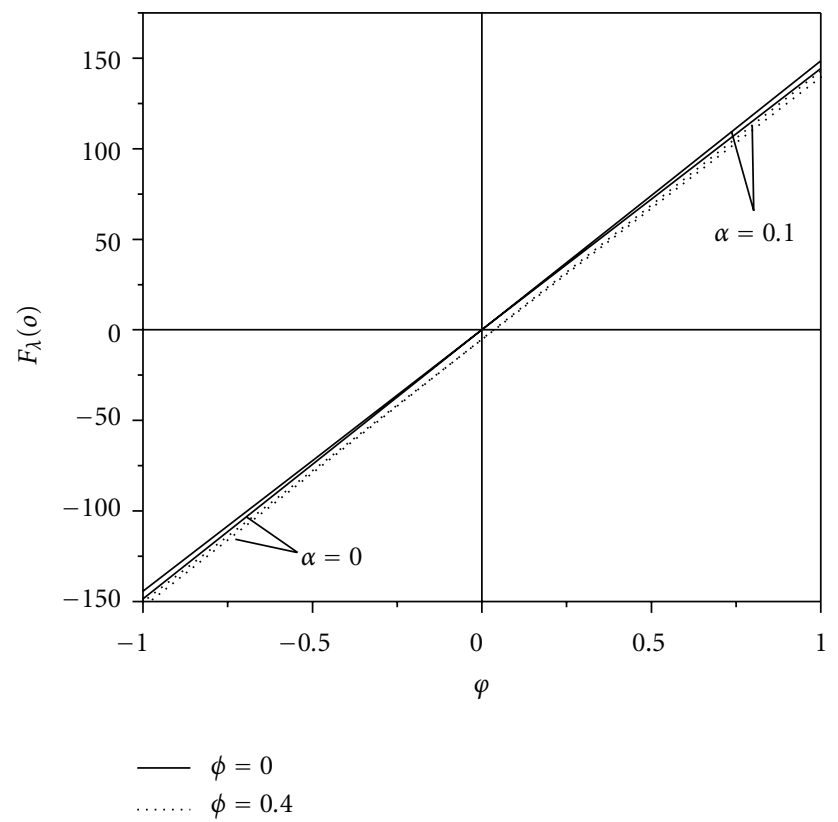

Figure 4: The friction force on the outer tube versus flow rate for $\varepsilon=0.32, \alpha=0.0, \alpha=0.1$. 


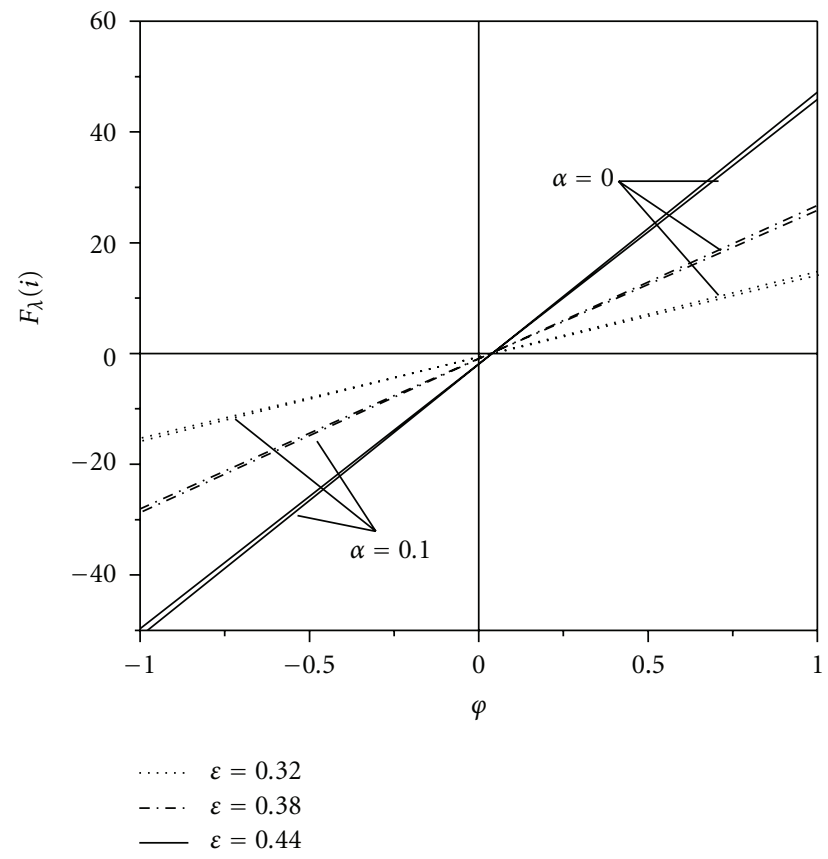

Figure 5: The friction on the inner tube (endoscope) versus flow rate for $\phi=0.2$, and $\alpha=0.0, \alpha=0.1$.

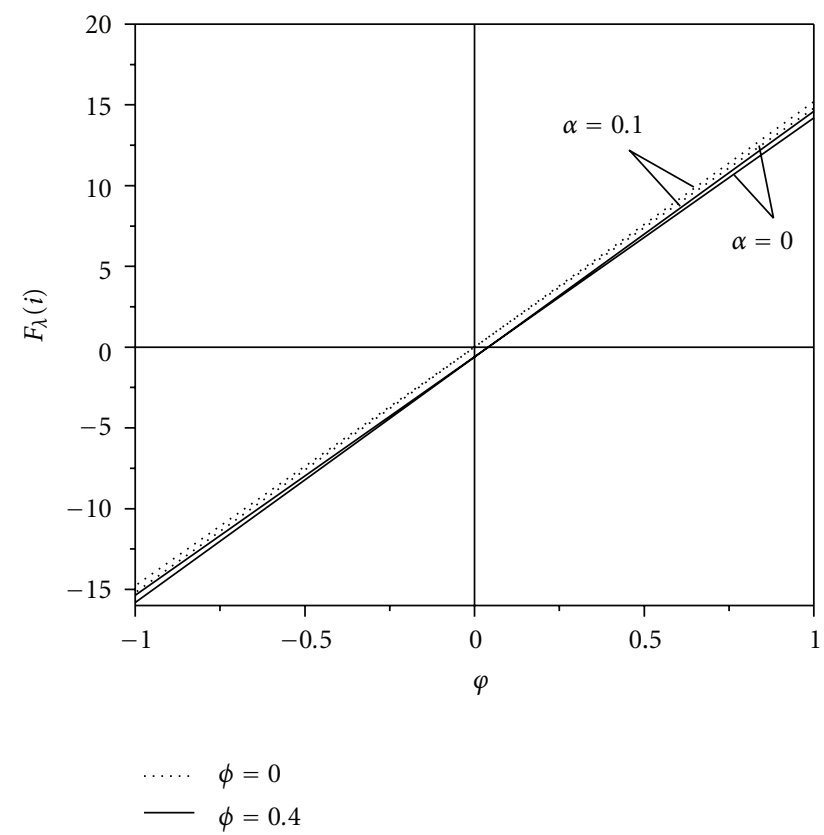

Figure 6: The friction on the inner tube (endoscope) versus flow rate for $\varepsilon=0.32, \alpha=0.0$, and $\alpha=0.1$. 


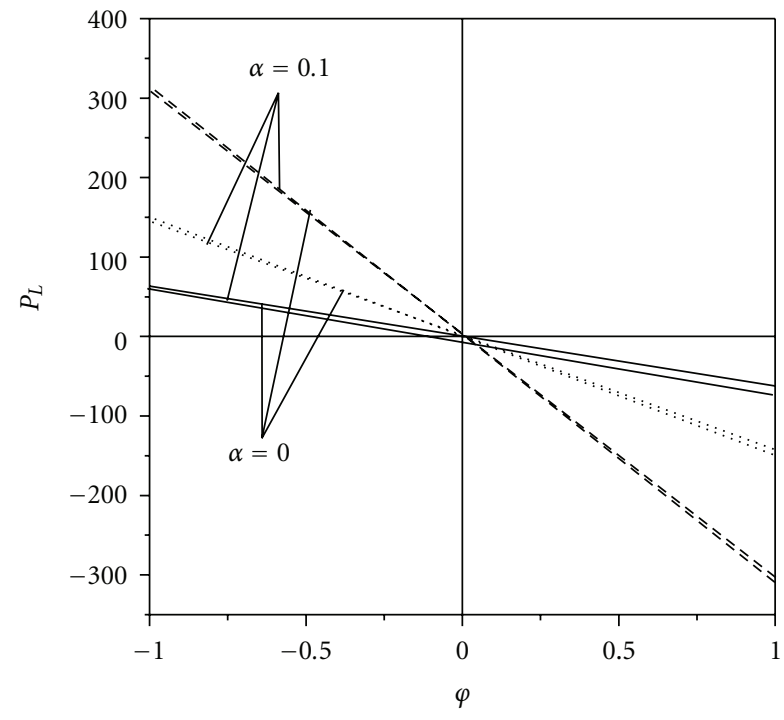

$\begin{aligned}- & M=1 \\ \cdots \cdots & M=3 \\ --- & M=5\end{aligned}$

Figure 7: The pressure versus flow rate for $\varepsilon=0.32, \alpha=0.0, \alpha=0.1$, and $\phi=0.2$.

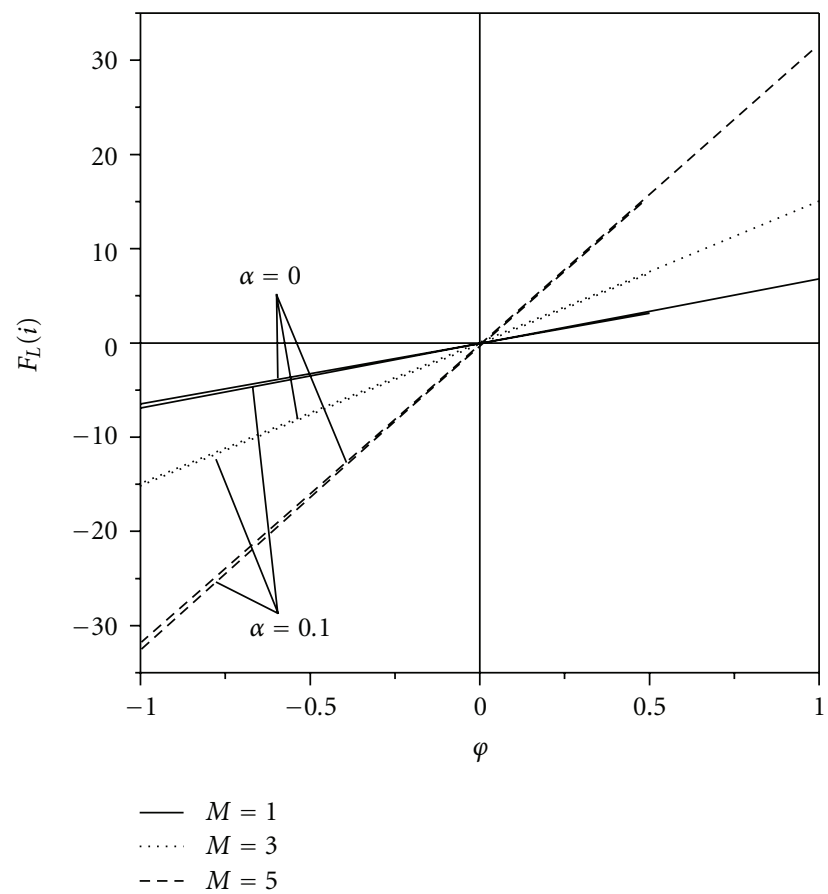

Figure 8: The friction on the inner tube (endoscope) versus flow rate for different magnetic field for $\phi=0.2$, $\varepsilon=0.32, \alpha=0.0$, and $\alpha=0.1$. 


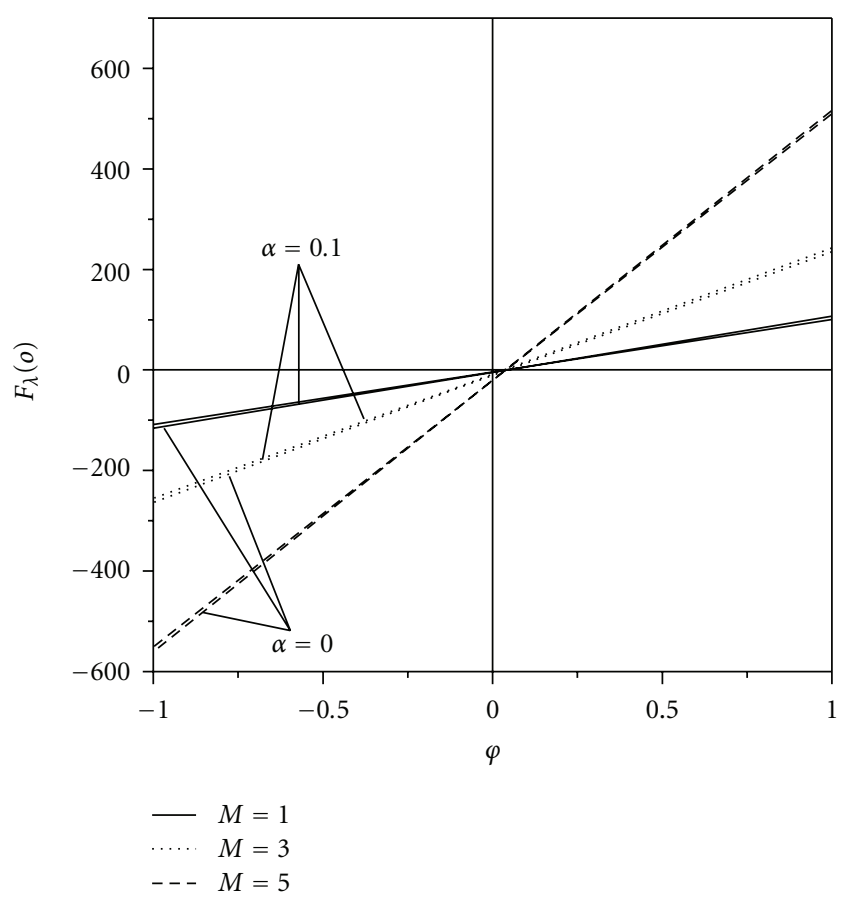

Figure 9: The friction on the outer tube versus flow rate for different magnetic field for $\varepsilon=0.32, \phi=0.2$, $\alpha=0.0$, and $\alpha=0.1$.

\section{References}

[1] L. M. Srivastava, V. P. Srivastava, and S. N. Sinha, "Peristaltic transport of a physiological fluid. Part I. Flow in non-uniform geometry," Biorheology, vol. 20, no. 2, pp. 153-166, 1983.

[2] A. M. Siddiqui and W. H. Schwarz, "Peristaltic flow of a second-order fluid in tubes," Journal of NonNewtonian Fluid Mechanics, vol. 53, pp. 257-284, 1994.

[3] T. W. Latham, M.S. thesis, MIT, Cambridge, Mass, USA, 1966.

[4] J. C. Burns and T. Parkes, "Peristaltic motion," Journal of Fluid Mechanics, vol. 29, pp. 731-743, 1967.

[5] C. Barton and S. Raynor, "Peristaltic flow in tubes," Bulletin of Mathematical Biophysics, vol. 30, p. 663, 1968.

[6] P. S. Lykoudis and R. Roos, "The fluid mechanics of the ureter from a lubrication theory point of view," Journal of Fluid Mechanics, vol. 43, p. 661, 1970.

[7] T.-F. Zien and S. Ostrach, "A long wave approximation to peristaltic motion," Journal of Biomechanics, vol. 3, no. 1, pp. 63-75, 1970.

[8] R. Roos and P. S. Lykoudis, "The fluid mechanics of the ureter," Journal of Fluid Mechanics, vol. 46, p. 625, 1971.

[9] R. A. Ramchandra and S. Usha, Biochemical Engineering Journal, vol. 117, p. 448, 1995.

[10] A. H. Abel El Naby and A. E. M. El Misery, "Effects of an endoscope and generalized Newtonian fluid on peristaltic motion," Applied Mathematics and Computation, vol. 128, pp. 19-35, 2001.

[11] K. K. Gupta and V. Sheshadri, "Peristaltic pumping in non-uniform tubes," Journal of Biomechanics, vol. 9, pp. 105-109, 1976.

[12] L. M. Srivastava and V. P. Srivastava, "Peristaltic transport of a non-newtonian fluid: applications to the vas deferens and small intestine," Annals of Biomedical Engineering, vol. 13, pp. 137-153, 1985.

[13] A. M. Provost and W. H. Schwarz, "Theoretical study of viscous effects in peristaltic pumping," Journal of Fluid Mechanics, vol. 279, pp. 177-195, 1994. 
[14] G. Boehme and R. Friedrich, "Peristaltic flow of viscoelastic liquids," Journal of Fluid Mechanics, vol. 128, pp. 109-122, 1983.

[15] A. M. El Misery, E. F. Elshehawey, and A. A. Hakeem, "Peristaltic motion of an incompressible generalized newtonian fluid in a planar channel," Journal of the Physical Society of Japan, vol. 65, no. 11, pp. 3524-3529, 1996.

[16] E. F. Elshehawey, A. E. M. El Misery, and A. E. H. Abd El Naby, "Peristaltic motion of generalized newtonian fluid in a non-uniform channel," Journal of the Physical Society of Japan, vol. 67, no. 2, pp. 434-440, 1998.

[17] R. H. Haynes, "Physical basis of the dependence of blood viscosity on tube radius," The American Journal of Physiology, vol. 198, pp. 1193-1200, 1960.

[18] G. Bugliarllo and J. Servilla, Biorheology, vol. 7, p. 85, 1970.

[19] H. L. Goldsmith and R. Skalak, "Hemodynamics," in Annual Review of Fluid Mechanics, Vol. 7, Palo Alto Publications 231, pp. 213-247, Annual Reviews, Palo Alto, Calif, USA, 1975.

[20] P. B. Cotton and C. B. Williams, Pratical Gasstrointestinal Endoscopy, chapter 2, Oxford Press, London, UK, 3rd edition, 1990.

[21] V. P. Rathod and S. K. Asha, "Peristaltic transport of a couple stress fluid in a uniform and non uniform annulus," International journal of mathematical modeling ,simulation and application, vol. 2, no. 4, pp. 414 426, 2009.

[22] V. P. Rathod and S. K. Asha, "Effect of couple stress fluid and an endoscope in peristaltic motion," Ultra Science, vol. 21, no. 1, pp. 83-90, 2009. 


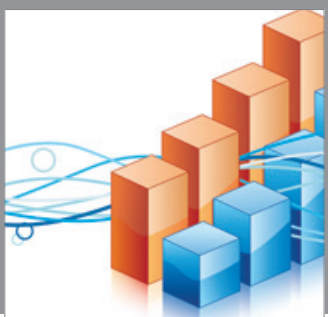

Advances in

Operations Research

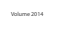

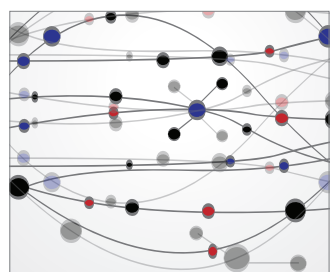

\section{The Scientific} World Journal
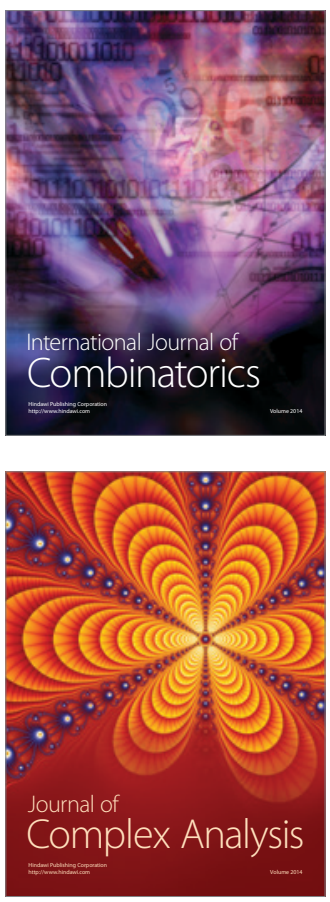

International Journal of

Mathematics and

Mathematical

Sciences
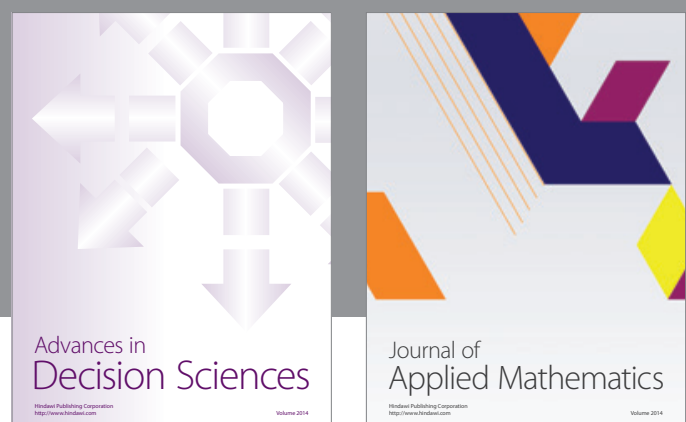

Journal of

Applied Mathematics
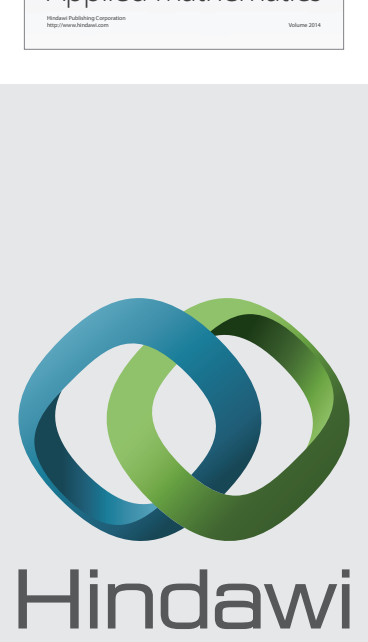

Submit your manuscripts at http://www.hindawi.com
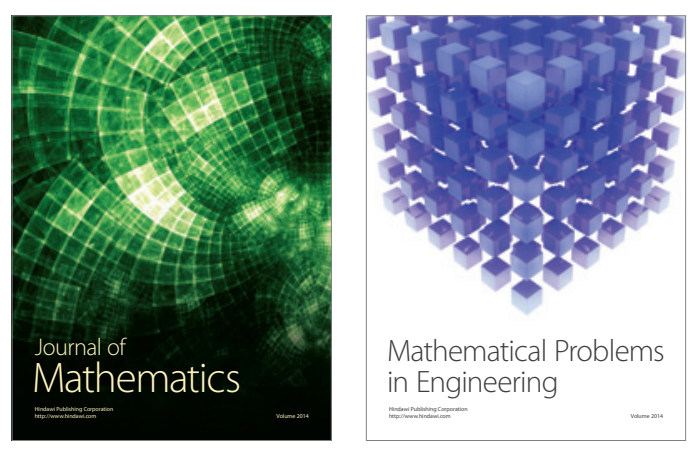

Mathematical Problems in Engineering
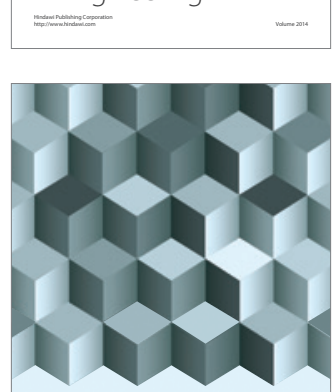

Journal of

Function Spaces
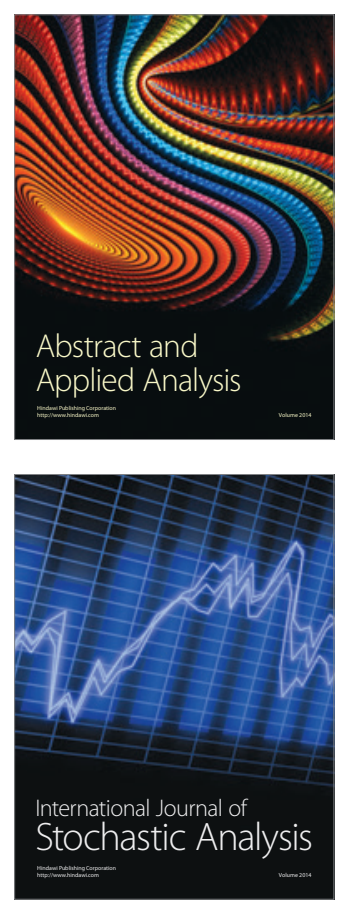

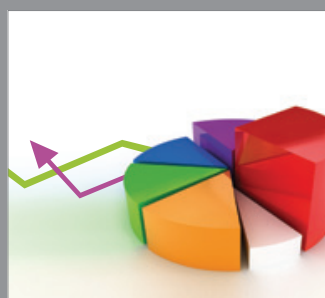

ournal of

Probability and Statistics

Promensencen
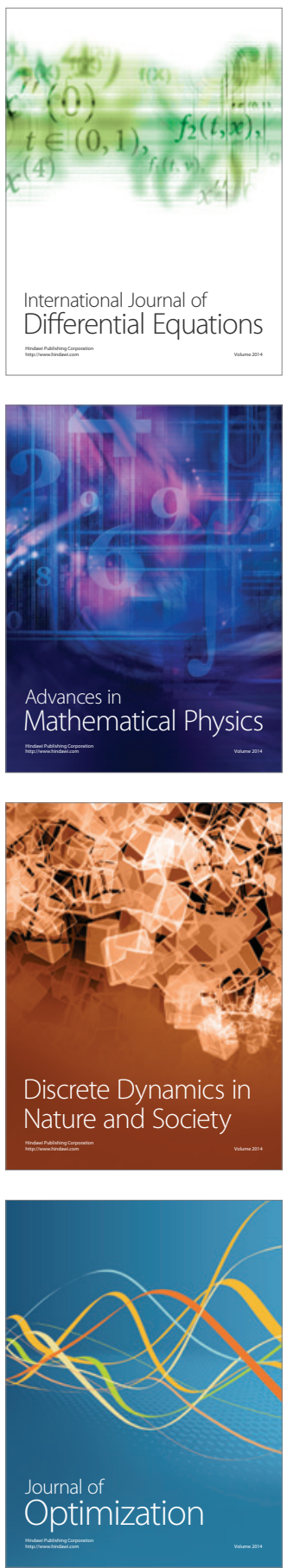